\title{
Gordan's Theorem for Double Binary Forms.
}

By Professor H. W. Turnaull.

(Received 18th November 1922. Read 9th February 1929).

\$1. Gordan's Theorem, that the complete system of irreducible concomitants of a given form is finite, has been extended by Hilbert * to cover wide ranges of systems of variables Gordan $\dagger$ and Study $\ddagger$ have dealt shortly with the problem for double binary forms, approaching the subject through the theory of binary types. The following pages give a proof after the manner of Gordan's proof for ordinary binary forms, which bas the advantage of providing a practical method for constructing the complete system. As illustrations, the cases of the $(1,2),(2,2)$, and $(3,3)$ forms are considered.

It is probable that the method would also solve the problem of double binary perpetuants. $\$$

§ 2. In the Proc. Roy. Soc. Edinburgh, Vol. xliii., 1923, I have discussed double binary forms, introducing the nomenclature of Gordan, necessary in the following proof which deals with the system belonging to the double binary $\left(n, n^{\prime}\right)$ form

$$
f=a_{z}^{n} a_{z^{\prime}}^{\prime n^{\prime}}=b_{z}^{n} b_{z^{\prime}}^{\prime n^{\prime}}=\text { etc. }
$$

If $z=x+i y, z^{\prime}=x-i y$ and $n=n^{\prime}$ then $f$ answers to plane circular curves. There appears to be no way of proving this theorem by an induction involving only symmetrical forms $(1,1),(2,2), \ldots(n, n)$; but the system $\left(n, n^{\prime}\right)$ can be found if $n^{\prime} \equiv n$ and if all systems $\left(N, n^{\prime}-i\right)$ are known where $i>0$ and $N$ is a finite number depending on $n, n^{\prime}$.

Suppose that the knowledge of the $\left(n, n^{\prime}\right)$ system requires a preliminary system copied from the complete system $(\lambda, \mu)$, then

* Malh. Ann., Bd. 30 and 36. Cf. Maurer, "Uuber die Endlichkeit der Invarianten Systeme." München. Sitzungsberichte der Math. Bd. 29, 1899.

† Cf. Math. Ann., Bd. 33, pp. 387.389; also Sitz. berich. der Phys.-med. Soc. Erlangen, (1888), p. 35.

$¥$ Ibid., p. 31 .

Of. Grace and Young, Algebra of Invariants, pp. 326-338. 
the $\left(n, n^{\prime}\right)$ system is said to involve the $(\lambda, \mu)$ system. It is frst necessary to prove a lemma to show that the number of systems involved in $\left(n, n^{\prime}\right)$ is finite, and that the systems can be arranged in a definite order. Let the system $\left(n-i, n^{\prime}-j\right)$ be called of lower rank if one or both of $i, j$ exceed zero.

§3. Lemma I. - If, for all values of $n, n^{\prime}$ such that $n \equiv n^{\prime}, n \geqslant n_{0}$, $n^{\prime} ₹ n_{0}^{\prime}$, the systems involved in $\left(n, n^{\prime}\right)$ are either of lower rank or are included in the systems $\left(k n, n^{\prime}-i\right), i=1,2 \ldots n^{\prime}$, then $\left(n_{0}, n_{0}{ }^{\prime}\right)$ innolves a finite number of systems and they can be ordinally arranged, $k$ being a finite positive integer.

For let $(\alpha, \beta)$ denote any one of the systems here specified; then $\beta \leq n_{0}{ }^{\prime}$, whereas a may exceed $n_{0}$, but certainly does not exceed $k^{n_{0}} . n_{0}$ which is finite. Corresponding to values of $\beta$ from 0 to $n_{0}{ }^{\prime}$ inclusive let the greatest values of $\alpha$ be $\alpha_{0}, \alpha_{1}, \ldots \alpha_{n_{0}^{\prime}}$. It follows that the systems involved in $\left(n, n^{\prime}\right)$ which are not of lower rank are included in those whose rank is lower than that of $\left(\alpha_{0}, 0\right)$, $\left(\alpha_{1}, 1\right), \ldots\left(\alpha_{n_{0}^{\prime}}, n_{0}^{\prime}\right)$. But this last sequence also includes systems of lower rank than $\left(n, n^{\prime}\right)$ since $a_{i} \geq n, k>0$. The sequence, therefore, defines the order required, namely the first $a_{0}$ binary forms in ascending order, followed by $\alpha_{1}$ forms linear in $z^{\prime}$ in ascending order of $z$, followed by $\left(\alpha_{2}-1\right)$ forms of the second order in $z^{\prime}$ and of orders $2,3, \ldots \alpha_{2}$ respectiveiy in $z$; and so on till $\alpha_{n / 8}$ is reached. It will be seen that $a_{n_{0}^{\prime}}=n_{0}$.

Since the systems $(n, m)$ and $(m, n)$ are of the same character, and one is known if the other is known, then there has been no loss of generality in the argument above by assuming $n \geq n^{\prime}$.

This lemma is required at several stages in the following proof of Gordan's Theorem, the value of $k$ being either 2 or 3 . It will be found that, in particular, knowledge of the binary duodecimic must precede that of the $(3,3)$ form.

\$4. Systems of forms derived by transvection from two given systems.

'Phe developments of the binary theory (Cf. Algebra of Invariants, loc. cit.) apply with a few modifications to the present case. 
Thus if $U, V$ are symbolic products $A_{1}^{\alpha_{1}} \ldots A_{m}^{\alpha_{m}}$ and $B_{1}^{\beta_{1}} \ldots B_{n}^{\beta n}$ of forms belonging to two systems $(A)$ and $(B)$, the set of terms of all possible transvectants

$$
\langle U, V)^{\rho \rho_{1}}
$$

is called the system derived by transvection from (A) and (B). Any such transvectant contains reducible terms if, when

$$
U=U_{1} U_{2}, V=\nabla_{1} V_{2}, \rho=\sigma+\tau, \rho_{1}=\sigma_{1}+\tau_{1},
$$

it is possible to construct $\left(U_{1}, V_{1}\right)^{\sigma \sigma_{1}}$ and $\left(U_{2}, V_{2}\right)^{\tau \tau_{1}}$; for example, $\sigma$ must not exceed the order of $z$ in $U_{1}$. It follows that the number of transvectants $(U, V)^{\rho \rho_{1}}$ (derived from finite systems $(A)$ and $(B)$ ) which do not contain reducible terms is finite. The proof is that for binary forms with four Diophantine equations in place of two : let this be called Lemma II.

It is convenient to have a symbol to denote systems derived by transvection as above. Let

$$
[(A),(B)]
$$

denote such a system. Then the notation may be extended to more complex systems as

$$
[[(A),(B)],(C)]
$$

The two further lemmas of the binary theorem may be taken over with little modification. Let $(A) \equiv c$, or $(A) \equiv c \bmod H$ symbolise that the system $(A)$ is finite and complete, or that the system $(A)$ is finite and relatively complete for the modulus $H$. Then we may enunciate the two lemmas :

Lrmma III. $-I f(A) \equiv c$ and $(B) \equiv c$, then $[(A),(B)] \equiv c$.

LEM IVA IV.-If a finite system of forms $(A)$, all the members of which are covariants of the $\left(n, n^{\prime}\right)$ form $f$, include $f$, and if $(A) \equiv c \bmod H, K$; if further $(B) \equiv c \bmod G$, where $(B)$ includes one form $B_{1}$ whose only determinantal factors are $H$, then the relation

is satisfied.

$$
[(A),(B)] \equiv c \bmod G, K
$$

These may be proved as for binary forms if we add the following modifications : transvectants should be considered :-

(i) In order of ascending total degree of $U V$ in the coefficients of the forms involved in $A, B$. 
(ii) Those for which the degree of $U V$ is the same are taken in ascending degree of $U$.

(iii) Those for which these two degrees are the same are taken in ascending order of the total index $\left(\rho+\rho_{1}\right)$.

\section{§5. Method of proof of Gordan's Theorem.}

If we can successively build up systems of

$$
f=a_{z}^{n} a_{z^{\prime}}^{n^{\prime}}=b_{z}^{n} b_{z^{\prime}}^{n^{\prime}}=\text { etc. }
$$

complete for the moduli $\left(a^{\prime} b^{\prime}\right),\left(a^{\prime} b^{\prime}\right)^{2}, \ldots\left(a^{\prime} b^{\prime}\right)^{n^{\prime}}$ respectively, then at the last stage the system is absolutely complete. Let such relative systems be called $A_{1}, A_{2}, \ldots A_{n^{\prime}}$. Then any form derived by convolution of members of the system $A_{r}$ is a rational integral function of these members or else contains a factor equivalent to $\left(a^{\prime} b^{\prime}\right)^{r}$. It must be recollected that $A_{r}$ involves any factor $(a b)$ to uny index up to $n$.

Let

$$
\begin{aligned}
H_{r u} & =(a b)^{r}\left(a^{\prime} b^{\prime}\right)^{r} a_{z}^{n-r} a_{z^{\prime}}^{n^{\prime}-s} b_{z}^{n-r} b_{z^{\prime}}^{, n^{\prime}-s} \\
h_{r u} & =(a b)^{r}\left(a^{\prime} b^{\prime}\right)^{s}:
\end{aligned}
$$

and let

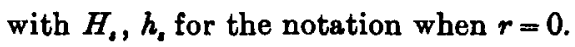

Then the construction of the systems $A_{1}, A_{2}, \ldots A_{n^{\prime}}$ falls into three sections according to the order of $H_{r a}$ in $z^{\prime}$, as 8 takes the values $1,2, \ldots n^{\prime}$ in succession.

First we consider $s<n^{\prime} / 2$, next $s=n^{\prime} / 2$, next $s>n^{\prime} / 2$.

\section{\$6. Case $I ., s<n^{\prime} / 2$.}

The system $A_{1}$ is the complete system of $f=a_{2}^{n} a_{z}^{n^{\prime}}$ regarded as a binary form of order $n$ in $z$. For if $C$ is a product of terms of this system then a convolution of $C$ contains at least one factor $\left(a^{\prime} b^{\prime}\right)$ or no factor $\left(a^{\prime} b^{\prime}\right)$ in which case the term is by hypothesis a function of terms $C$. Symbolically,

therefore

$$
\begin{aligned}
& \bar{C} \equiv W^{\prime}(C) \bmod \left(a^{\prime} b^{\prime}\right), \\
& C \equiv c \operatorname{modd}(a b)\left(a^{\prime} b^{\prime}\right),\left(a^{\prime} b^{\prime}\right)^{2} .
\end{aligned}
$$

Also $C$ includes $f$ and so satisfies the conditions for $A_{1}$.

Now suppose that we know all the systems $A_{1}, A_{2}, \ldots$ to $A_{1}$. We must form the next system $A_{s+1}$. There are two sub-cases 
according as $s=2 k-1$ or $2 k$. First if $8=2 k-1$; then we have

$$
\begin{aligned}
A_{d} & =A_{2 k-1} \equiv c \bmod \left(a^{\prime} b^{\prime}\right)^{2 k-1} \\
& \equiv c \operatorname{modd}\left(a^{\prime} b^{\prime}\right)^{2 k-1}(a b),\left(a^{\prime} b^{\prime}\right)^{2 k} .
\end{aligned}
$$

Now we can shew that if $B_{k}$ is the auxiliary system consisting of the single form

$$
K=H_{1,2 k-1}=(a b)\left(a^{\prime} b^{\prime}\right)^{2 k-1} a_{z}^{n-1} b_{z}^{n-1} a_{z^{\prime}}^{\prime \prime 2 k+1} b_{z^{\prime}}^{\prime n !-2 k+1},
$$

then $B_{k}$ is complete for the modulus $\left(a^{\prime} b^{\prime}\right)^{2 k}$. This follows if we can shew that all transvectants

$$
(K, K)^{i i_{1}}
$$

are of grade $2 k$ in $\left(a^{\prime} b^{\prime}\right)$. But if $i_{1} \neq 0$ this last transvectant may be dealt with as a binary form in $a^{\prime} b^{\prime} c^{\prime} d^{\prime}$ which is known to be of higher grade since $2 k-1=s<\frac{n^{\prime}}{2}$ (Cf. Algebra of Invariants, pp. 71-77). Also when $i_{1}=0$ each term of this transvectant is of type

$$
\left(a^{\prime} b^{\prime}\right)^{2 k-1}\left(c^{\prime} d^{\prime}\right)^{2 k-1}(a b)(c d) M .
$$

But after bracketing $a^{\prime}$ in each of the $2 k-1$ brackets $\left(c^{\prime} a^{\prime}\right)$, this type may be expressed as a sum of transvectants $N(\theta, \delta)^{\mu}$ where $\delta$ contains all the symbols $d^{\prime}$ and $\theta$ all $a^{\prime} b^{\prime} c^{\prime}$ and $N$ all symbols belonging to $z$. In this, $\theta$ is of higher grade $2 k$ (loc cit. $\$ 70)$ since the bracket factors of $\theta=\left(a^{\prime} b^{\prime}\right)^{2 k-1}\left(a^{\prime} c^{\prime}\right)^{\lambda}$ and $2 k-1 \geq \lambda>\frac{1}{2}(2 k-1)$.

Hence $B_{k}$ satisfies the relation

$$
B_{k} \equiv c \bmod \left(a^{\prime} b^{\prime}\right)^{2 k},
$$

whence, by Lemma IV,

$$
\left[A_{2 k-1}, B_{k}\right] \equiv c \bmod \left(a^{\prime} b^{\prime}\right)^{2 k}
$$

which determines $A_{2 k}$, that is $A_{s+1}$, if $s$ is odd.

Next if $s=2 k$ we must find the system $A_{2 k+1}$ from $A_{2 k}$. We shall require an auxiliary system $B_{2 k}$ containing $\left(a^{\prime} b^{\prime}\right)^{2 k}\left(=h_{2 k}\right)$ such that

$$
B_{2 k} \equiv c \bmod \left(a^{\prime} b^{\prime}\right)^{2 k+1} \text {. }
$$

Suppose that the binary $(n, 0)$ system $A_{1}$ is

$$
(\phi)=\phi_{1}, \phi_{2}, \ldots \phi_{p},
$$

each $\phi$ being a generalized transvectant of $f$. Since $h_{2 k}$ denotes $\left(a^{\prime} b^{\prime}\right)^{2 k}$ the orders $\left(2 n, 2 n^{\prime}-2 k\right)$ of $H_{2 k}$ are of higher rank than 
$(n, 0)$ respectively. So we may copy a set of transvectants for $H_{2 k}$,

$$
(\psi)=\psi_{1}, \psi_{2}, \ldots \psi_{p},
$$

on the model of the set $(\phi)$ of lower rank.

If $\left(\overline{H_{2 k}}, \overline{H_{2 k}}, \ldots\right)$ denote a generalized transvectant of forms $\overline{H_{2 k}}$, where at least one of $\overline{H_{2 k}}$, is convolved from $H_{2 k}$ and the others are identical with $H_{2 k}$, then any term $t_{r}$ of $\psi_{r}$ may be written

$$
\left.t_{r}=\psi_{r}+\Sigma \overline{\left(H_{2 k}\right.}, \overline{H_{2 k}}, \ldots\right) \text {. }
$$

But, for the convolved term, $\overline{H_{2 k}} \equiv 0$ modd $\left(a^{\prime} b^{\prime}\right)^{2 k+1},\left(a^{\prime} b^{\prime}\right)^{2 k}(a b)$; hence $\quad t_{r} \equiv \psi_{r} \operatorname{modd}\left(a^{\prime} b^{\prime}\right)^{2 k+1},\left(a^{\prime} b^{\prime}\right)^{2 k}(a b)$.

Again, since the system $(\phi)$ is absolutely complete, then any term convolved from a product of $(\psi)$ must satisfy

$$
\bar{\psi} \equiv F(\psi) \bmod Q
$$

where $Q$ denotes transvectants whose index for $a^{\prime}$ symbols exceeds zero, otherwise $Q$ is within the range copied from the $(n, 0)$ form and therefore included in $F(\psi)$.

But, if $i>0,2 k<\frac{n^{\prime}}{2}$,

$$
\left.\left(a^{\prime} b^{\prime}\right)^{2 k}\left(b^{\prime} c^{\prime}\right)^{i}\left(c^{\prime} d^{\prime}\right)^{2 k} \equiv 0 \bmod \left(a^{\prime} b^{\prime}\right)^{2 k+1} \quad \text { (loc. cit. } \$ 73\right),
$$

then hence if $r_{2}>0,\left(H_{2,, 2 k}, H_{2 q_{2} 2 k}\right)^{r_{1}} r_{2} \equiv 0 \bmod \left(a^{\prime} b^{\prime}\right)^{2 x+1}$.

This implies that $Q$ is of grade $\left(a^{\prime} b^{\prime}\right)^{2 k+1}$. It follows that the system

$$
\left(t_{1}=t_{1}, t_{2}, \ldots t_{p}\right.
$$

includes $H_{2 k}$ and is complete for the moduli $\left(a^{\prime} b^{\prime}\right)^{2 k}(a b),\left(a^{\prime} b^{\prime}\right)^{2 k+1}$ whose total indices are odd. Hence

so that

$$
(t)_{1} \equiv c \operatorname{modd}\left(a^{\prime} b^{\prime}\right)^{2 k}(a b)^{2},\left(a^{\prime} b^{\prime}\right)^{2 k+1}
$$

$$
\left[A_{2 k},(t)_{1}\right] \equiv c \text { modd }\left(a^{\prime} b^{\prime}\right)^{2 k}(a b)^{\prime},\left(a^{\prime} b^{\prime}\right)^{2 k+1} \text {. }
$$

Proceeding in the same way we may rid ourselves of the first of these two moduli by forming a system $(t)_{2}$ for the $\left(2 n-4,2 n^{\prime}-4 k^{\prime}\right)$ form

$$
H_{2,2 k}=\left(a^{\prime} b^{\prime}\right)^{2 k}(a b)^{2} Z,
$$

$Z$ representing necessary $a_{z} a_{z^{\prime}}^{\prime}$ factors, on the model of

$$
(\phi)=\phi_{1}, \phi_{2}, \ldots \phi_{p} \text {, }
$$


since the orders of $H_{2.22}$ both exceed $(n, 0)$. Then

$$
(t)_{2} \equiv c \text { modd }\left(a^{\prime} b^{\prime}\right)^{2 k}(a b)^{4},\left(a^{\prime} b^{\prime}\right)^{2 k+1} .
$$

Similarly the systems

$$
\begin{aligned}
& (t)_{2} \equiv, \quad, \quad, \quad(a b)^{6}, \quad, \quad, \\
& \ldots \ldots \ldots \ldots \ldots \ldots \ldots \ldots \ldots \ldots \ldots \ldots \ldots \ldots \ldots \ldots \ldots \ldots \ldots \ldots \ldots \ldots \ldots \ldots
\end{aligned}
$$

may be derived from $H_{4,2 k}, \ldots H_{2 q-2,2 k}$, provided the orders of each $H$ exceed or equal $(n, 0)$. The limit is reached when

If now

$$
2 q=\frac{n}{2} \text { or } \frac{n-1}{2}
$$

$$
{ }_{q} A_{2 k}=\left[\ldots\left[\left[A_{2 k},(t)_{1}\right],(t)_{2}\right], \ldots,(t)_{q}\right]
$$

denote successive transvections employing $(t)_{1} \ldots(t)_{q}$, then ${ }_{q} A_{2 k}$ will be finite and complete for the moduli of $(t)_{q}$, that is

$$
{ }_{4} A_{2 k} \equiv c \operatorname{modd}\left(a^{\prime} b^{\prime}\right)^{2 k}(a b)^{2 q},\left(a^{\prime} b^{\prime}\right)^{2 k+1} .
$$

This is a half-way stage between $A_{8}$ and $A_{t+1}$. To proceed. The next value of $q$ gives $2 q>\frac{n}{2}$ so that the orders of $H_{2 q, 2 k}$ are $\left(2 n-4 q, 2 n^{\prime}-4 k^{\prime}\right)$ or $\left(r, r^{\prime}\right)$ say, where $r<n$. For a model take the complete system

$$
(\chi)=\chi_{1}, \chi_{2}, \ldots \chi_{\omega}
$$

of the $(r, 0)$ form which is known by hypothesis, and let the copied system of $H_{2 g, 2 k}$ be $\left(\chi^{\prime}\right)$. The same argument will now apply again.

Thus single terms one from each $\chi^{\prime}$ are complete for the modulus $\left(a^{\prime} b^{\prime}\right)^{2 k+1}$ and a modulus due to $\bar{H}$ as before, giving

$$
(t)_{g} \equiv c \text { modd }\left(a^{\prime} b^{\prime}\right)_{-}^{2 k}(a b)^{24},\left(a^{\prime} b^{\prime}\right)^{2 k+1},
$$

for all values of $2 q>\frac{n}{2}$ up to $2 q=n$ or $n-1$ according as $n$ is even or odd. For the next value of $q$ the former modulus does not exist; the corresponding system $(t)$ may be taken as $B_{2 k}$ and we shall bave

$$
\left[A_{2 k}, B_{2 k}\right] \equiv c \bmod \left(a^{\prime} b^{\prime}\right)^{2 k+1}, q=\frac{n}{2} \text { or } \frac{n-1}{2} .
$$

This is the system $A_{2 k+1}$ or $A_{t+1}$ when 8 is even. 
§7. Case $I I ., 8=\frac{n^{\prime}}{2}$.

As in Algebra of Invariants, $\$ 73$, this case leads to a new modulus when $s$ is even: the system derived from $A$, by the preceding argument will be finite and complete for moduli

$$
\left(a^{\prime} b^{\prime}\right)^{s+1},\left(b^{\prime} c^{\prime}\right)^{\prime}\left(c^{\prime} a^{\prime}\right)^{s}\left(a^{\prime} b^{\prime}\right)^{\prime} .
$$

Let this system be called $C_{a}$. To derive the system $A_{a+1}$ from $C$, we proceed as follows:-

Let

$$
\begin{aligned}
J & =\left(b^{\prime} c^{\prime}\right)^{a}\left(c^{\prime} a^{\prime}\right)^{a}\left(a^{\prime} b^{\prime}\right)^{s} a_{z}^{n} b_{z}^{n} c_{z}^{n} \\
j & =\left(b^{\prime} c^{\prime}\right)^{*}\left(c^{\prime} a^{\prime}\right)^{\prime}\left(a^{\prime} b^{\prime}\right)^{s}, \\
(\Omega) & =\Omega_{1}, \Omega_{2}, \ldots \Omega_{i}
\end{aligned}
$$

and suppose

the complete system of the binary form $J$ of orders $(3 n, 0)$ which by Lemma $I$. is already known. As before let

$$
(\tau)_{0}=\tau_{1}, \tau_{2}, \ldots \tau_{i}
$$

denote single terms chosen from $(\Omega)$, each $\Omega$ being given as a generalized transvectant of $J$; then

$$
\tau_{r} \equiv \Omega_{r}+\Sigma(\bar{J}, \bar{J}, \ldots)
$$

Since $(\Omega)$ is absolutely complete, then

where

$$
(\tau)_{0} \equiv c \bmod \bar{J}
$$

$$
\bar{J}=j(b c)^{p}(c a)^{q}(a b)^{r} Z, p+q+r>0 ;
$$

and since $j$ is of weight $3 s$ which is even, we may take $p+q+r>1$ and so, by Jordan's Lemma, at least one of $p, q, r$ may be taken as not less than 2. This Lemma states that if $x+y+z=0$, then any product of powers of $x, y, z$ of order $m$ can be expressed linearly in terms of such products as contain one exponent equal to or greater than $\frac{2 m}{3}$. It applies here since

$$
\text { (bc) } a_{z}+(c a) b_{z}+(a b) c_{z}=0,
$$

and it may be further applied to the case above where $p+q+r=m$ so as to express all moduli $(b c)^{p}(c a)^{g}(a b)^{r}$ in terms of a smaller set of the same type with $p \geq \frac{2 m}{3}$. Let such a set, when each member 
is multiplied by $j$, be written

$$
(j)_{m}=j_{m}^{\prime}, j_{m}^{\prime \prime}, \ldots j_{m}^{(k m)} \text {. }
$$

We may therefore construct a sequence of systems $\Delta_{0}, \Delta_{2} \ldots \triangle_{m}$ by introducing systems $(\tau)_{m}^{\prime},(\tau)_{m}^{\prime \prime}, \ldots$ each from binary forms belonging to $j_{m}^{\prime}, j_{m}^{\prime \prime}, \ldots$ exactly as $(\tau)_{0}$ was constructed from $j$. Thus

$$
\begin{aligned}
& \triangle_{0}=\left[C_{k},(\tau)_{0}\right] \equiv c \operatorname{modd}\left(a^{\prime} b^{\prime}\right)^{k+1}, j_{2}, \\
& \triangle_{2}=\left[\triangle_{0},(\tau)_{2}\right] \equiv c \text { modd }\left(a^{\prime} b^{\prime}\right)^{k+1},(j)_{4} ;(j)_{4}=j_{4}^{\prime \prime}, j_{4}^{\prime \prime} ; \\
& \triangle_{4}=\left[\left[\triangle_{2},(\tau)_{4}^{\prime}\right],(\tau)_{4}^{\prime \prime}\right] \equiv c \operatorname{modd}\left(a^{\prime} b^{\prime}\right)^{k+1},(j)_{6} \\
& \Delta_{m}=\left[\ldots\left[\Delta_{m-2},(\tau)_{m}^{\prime}\right] \ldots(\tau)_{m}^{(k m)}\right] \\
& \equiv c \operatorname{modd}\left(a^{\prime} b^{\prime}\right)^{k+1},(j)_{m+2}, m=\frac{3 n}{2}-2 .
\end{aligned}
$$

Finally when $m=\frac{3 n}{2}-2,(j)_{m+2}$ consists of the single invariant*

$$
I=[(b c)(c a)(a b)]^{\frac{n}{2}}\left[\left(b^{\prime} c^{\prime}\right)\left(c^{\prime} a^{\prime}\right)\left(a^{\prime} b^{\prime}\right)\right]^{\frac{n}{2}} .
$$

If $I$ is added to the system $\triangle_{m}$, the combination will be com plete for the modulus $\left(a^{\prime} b^{\prime}\right)^{k+1}$, which is what we require Thus we have found a system $A_{a+1}$ from $A_{a}$ if either $8<\frac{n^{\prime}}{2}$ or $s=\frac{n^{\prime}}{2}$.

\$8. Case $I I I ., 8>\frac{n^{\prime}}{2}$.

Since $2 n^{\prime}-2 s<n^{\prime}$ we know by Lemma I. the complete system $(\Omega)_{k s}$ of the $\left(2 n-2 k, 2 n^{\prime}-2 s\right)$ form $H_{k s}$. Now the bracket factors of $H_{k,}$ are

$$
h(k, 8)=(a b)^{k}\left(a^{\prime} b^{\prime}\right)^{4} ;
$$

hence a form convolved from $\boldsymbol{H}_{k s}$ has bracket factors $h(p, q)$, where

$$
p \geq k, q \geq s, p+q>k+8 .
$$

As before let one term from each of the transvectants $(\Omega)_{k}$ be chosen and let $(\omega)_{k \text { s }}$ denote such chosen terms. Then, since $\Omega$ is absolutely complete,

$$
(\omega)_{k s} \equiv c \bmod h(p, q) \equiv c \operatorname{modd} h(k, s+1), h(k+1, s) .
$$

\footnotetext{
- $I=0$ unless $n, n^{\prime}, \frac{1}{2}\left(n+n^{\prime}\right)$ are all even.
} 
The former of these moduli is $\left(a^{\prime} b^{\prime}\right)^{a+1}$. Thus we may construct systems $E$, such that

$$
\begin{aligned}
& E_{1}=\left[A_{s},(\omega)_{0 . \Omega}\right] \equiv c \operatorname{modd}\left(\alpha^{\prime} b^{\prime}\right)^{s+1}, h(l, s), \\
& \dot{E_{2}}=\left[E_{1},(\omega)_{1 .}\right] \equiv, \quad, \quad, \quad, h(2, s) \text {, } \\
& E_{k+1}=\left[E_{k},(\omega)_{k, \text { l }}\right] \equiv, \quad, \quad, \quad, h(k+1, s) .
\end{aligned}
$$

Since $h(n+1, s)$ is identically zero, the system $E_{n}$ includes $A$, and is complete for the modulus $\left(a^{\prime} b^{\prime}\right)^{s+1} . E_{n}$ is therefore the required system $A_{i+1}$.

Thus we have shown that proceeding from $f=a_{z}^{n} a_{z^{\prime}}^{n !}$ successive systems $A_{1}, A_{2}, \ldots A_{a}$ can be found which are finite and complete for the successive moduli $\left(a^{\prime} b^{\prime}\right)^{i} i=1,2, \ldots s$. Since the system $A$, does not exist if $8>n^{\prime}$ the final system so found is absolutely complete, and Gordan's Theorem is therefore established.

§ 9. Application to the $(2,1)$ form.

Let $f=a_{x}^{2} a_{y}^{\prime}=b_{x}^{2} b_{y}^{\prime}$ be the $(2,1)$ form. Then $A_{1}$ is the system for the quadratic in $x$, and is

$$
f,(f, f)^{20} \text { or } H \text {. }
$$

This system being complete for $\left(a^{\prime} b^{\prime}\right)$ is complete for $(a b)\left(a^{\prime} b^{\prime}\right)$ since $\left(a^{\prime} b^{\prime}\right)^{2}$ does not exist.

But the system of $H_{11}=(a b)\left(a^{\prime} b^{\prime}\right) a_{x} b_{x}^{\prime}$ is again two forms

$$
H_{11},\left(H_{11}, H_{11}\right)^{20} \text { or } \triangle
$$

which is absolutely complete. Hence the complete system of $f$ is

$$
\left[A_{1}, B\right] \text { where } A_{1}=f, H \text {, and } B=H_{11}, \triangle .
$$

Only one new irreducible form appears by transvection in $[A, B]$, namely $\left(f H_{11}\right)^{10}$. So the complete system is *

$$
f, H, \triangle, H_{11},\left(f H_{11}\right)^{10} \text {. }
$$

" Of. Peano, Giornale di Math., Battaglini, Vol. xx., who reaches this result by elementary methods. The trestment of the $(2,2)$ form is very thorough. 
$\S$ 10. The $(2,2)^{*}$ form $f=a_{x}^{2} a_{y}^{\prime 2}=b_{x}^{2} b_{y}^{\prime 2}=$ etc.

The general method readily establishes the complete system of 18 forms. For the system $A_{1}$ consists of $f$ and $P_{2}$ where

$$
\begin{aligned}
& P_{2}=(a b)^{2} a_{y}^{\prime 2} b_{y}^{\prime 2} \\
& \text { and } \quad A_{1} \equiv c \bmod (a b)\left(a^{\prime} b^{\prime}\right),\left(a^{\prime} b^{\prime}\right)^{2} .
\end{aligned}
$$

Let $J=(a b)\left(a^{\prime} b^{\prime}\right) a_{x} b_{x} a_{y}^{\prime} b_{y}^{\prime}$ which is complete for the modulus $\left(a^{\prime} b^{\prime}\right)^{2}$. Then $\left[A_{1}, J\right] \equiv c \bmod \left(a^{\prime} b^{\prime}\right)^{2}$.

Since $f, J$ are of the same orders we need only consider the terms $(f, J)^{\rho \rho_{1}},\left(P_{2}, J\right)^{\rho \rho_{1}},\left(P_{2}, J^{2}\right)^{\rho \rho_{1}}, \rho, \rho_{1}=0,1,2$.

Since

$$
\begin{aligned}
J^{3} & =H f^{2}-2 C_{3} f+P_{2} P_{2}^{\prime}{ }^{*} \\
& =0 \operatorname{modd} f,\left(a^{\prime} b^{\prime}\right)^{2}
\end{aligned}
$$

the third of these transvectants may be rejected.

This gives the system

$$
A_{2}=f, P_{2}, J, P_{3}, \triangle \text {, }
$$

where

$$
\begin{aligned}
& A_{2} \equiv c \bmod \left(a^{\prime} b^{\prime}\right)^{2}, \\
& P_{\mathrm{s}}=(a b)^{2}\left(a^{\prime} c^{\prime}\right) c_{x}^{2} a_{y}^{\prime} b_{y}^{\prime 2} c_{y}^{\prime}, \\
& \triangle=(b c)(c a)(a b)\left(b^{\prime} c^{\prime}\right)\left(c^{\prime} a^{\prime}\right)\left(a^{\prime} b^{\prime}\right) .
\end{aligned}
$$

Now the modulus $\left(a^{\prime} b^{\prime}\right)^{2}$ of the system $A_{2}$ belongs to the binary quartic

$$
P_{2}^{\prime}=\left(a^{\prime} b^{\prime}\right)^{2} a_{x}^{2} b_{x}^{2}
$$

whose complete system is known. We may take single terms to represent members of this system, for such single terms are complete for modulus $M$, where $M$ is convolved from $P^{\prime}{ }_{2}$, that is

$$
M=\left(a^{\prime} b^{\prime}\right)^{2}(a b)=0 \text { or else } M=\left(a^{\prime} b^{\prime}\right)^{2}(a b)^{2}=H, \text { an invariant. }
$$

Let the single terms chosen be

$$
\begin{aligned}
P_{2}^{\prime}, Q_{4}^{\prime} & =\left(a^{\prime} b^{\prime}\right)^{2}(b c)^{2}\left(c^{\prime} d^{\prime}\right)^{2} Z, \\
P_{6}^{\prime} & =\left(a^{\prime} b^{\prime}\right)^{2}\left(c^{\prime} d^{\prime}\right)^{2}\left(e^{\prime} f^{\prime}\right)^{2}(b c)^{2}(d e), \\
\triangle_{4} & =(a b)^{2}(c d)^{2}\left(a^{\prime} d^{\prime}\right)^{2}\left(b^{\prime} c^{\prime}\right)^{2}, \\
J_{6} & =\left(a^{\prime} b^{\prime}\right)^{2}\left(c^{\prime} d^{\prime}\right)^{2}\left(e^{\prime} f^{\prime}\right)^{2}(b c)^{2}(d e)^{2}(a f)^{2} .
\end{aligned}
$$

and

Then if to these we add $H$ the whole set is absolutely complete.

Hence the compiete system of $f$ is the derived system of

$$
\left(f, P_{2}, P_{3}, J, \triangle\right) \text { and }\left(P_{2}^{\prime}, Q_{4}^{\prime}, P_{6}^{\prime}, \triangle_{4} B, J_{6}\right) \text {. }
$$

* Proc. Roy. Soc. Edinburgh. Vol. xliii., p. 50. 
The detailed investigation of these presents no serious difficulty. The indices of necessary transvectants never exceed 2 in value ; $J_{6}$ is reducible; $\triangle, \triangle_{4}, H$ are invariants. The result is a set of 18 forms.

\section{\$11. The $(3,3)$ form $f=a_{x}^{3} a_{y}^{\prime 3}$.}

Using the same notation as far as bracket factors are concerned $P_{2}^{\prime}$ is now a $(6,2)$ form $\alpha_{x}^{6} \alpha_{y}^{\prime 2}$. If $A_{1}$ is the system of $P_{2}^{\prime}$ as a binary sextic it is complete $\bmod \left(\alpha^{\prime} \beta^{\prime}\right)$, and therefore also complete modd $\left(\alpha^{\prime} \beta^{\prime}\right)(\alpha \beta),\left(\alpha^{\prime} \beta^{\prime}\right)^{2}$.

If now $J_{\alpha}=(\alpha \beta)\left(\alpha^{\prime} \beta^{\prime}\right) \alpha_{x}^{5} \beta_{x}^{5} \alpha_{y}^{\prime} \beta_{y}^{\prime}$, then $J_{\alpha} \equiv c \bmod \left(\alpha^{\prime} \beta^{\prime}\right)^{2}$. Hence $\left[A_{1}, J_{a}\right] \equiv c \bmod \left(\alpha^{\prime} B^{\prime}\right)^{2}$.

Now $\left(\alpha^{\prime} \beta^{\prime}\right)^{2} \alpha_{x}^{6} \beta_{x}^{6}$ is a binary form of order twelve. Single terms extracted from its complete system are complete mod $(\alpha \beta)^{2}\left(\alpha^{\prime} \beta^{\prime}\right)^{2}$. Transvecting these terms with $\left[A_{1}, J_{a}\right]$ produces a system complete $\bmod \left(\alpha^{\prime} B^{\prime}\right)^{2}(\alpha \beta)^{4}$.

This last modulus belongs to a binary quartic whose complete system together with the invariant $\left(\alpha^{\prime} \beta^{\prime}\right)^{2}(\alpha \beta)^{6}$ gives, by transvection with the existing system, a complete syst $\in \mathbf{m}$ for $P_{2}^{\prime}$.

Now take $f=a_{x}^{3} a_{y}^{\prime 3}$. From $f$ as a binary cubic in $x$ we construct $A_{1}=f, P_{2}, t, \triangle$ where $t=\left(P_{2}, f\right)^{10}, \triangle=\left(P_{2}, P_{2}\right)^{20}$.

If $J=(a b)\left(a^{\prime} b^{\prime}\right) a_{x}^{2} b_{x}^{2} a_{x}^{\prime 2} b_{x}^{\prime 2},\left[A_{1}, J\right] \equiv c \bmod \left(a^{\prime} b^{\prime}\right)^{2}(\S 6)$. But $P_{2}^{\prime}$ the $(6,2)$ form gives a system $B_{2}$ of single terms complete modd $\left(a^{\prime} b^{\prime}\right)^{3}(a b),\left(a^{\prime} b^{\prime}\right)^{2}(a b)^{2}$, of which the latter leads to a $(2,2)$ form whose system is complete in single terms $\bmod \left(a^{\prime} b^{\prime}\right)^{3}(a b)^{2}$, i.e. $\bmod \left(a^{\prime} b^{\prime}\right)^{3}(a b)^{3}$.

We can now deduce the system $A_{3}$ complete $\bmod (a b)^{3}(a b)$, and from the system of this modulus, i.e. from the binary quartic $\left(a^{\prime} b^{\prime}\right)^{3}(a b) a_{x}^{2} b_{x}^{2}$ we finally derive a complete system of $f$, if we include the invariant $\left(a^{\prime} b^{\prime}\right)^{3}(a b)^{3}$.

I take this opportunity of thanking the Committee of the Carnegie Trust for their material aid in furthering the preparation and the publication of this investigation. 